\title{
COMPARAÇÃO ENTRE METODOLOGIAS USEPA E IPCC PARA ESTIMATIVA TEÓRICA DE PRODUÇÃO DE BIOGÁS EM ATERRO MUNICIPAL
}

\author{
COMPARISON BETWEEN USEPA AND IPCC METHODOLOGIES FOR THEORETICAL \\ ESTIMATION OF BIOGAS PRODUCTION IN LANDFILL
}

\author{
Julia Bianek ${ }^{1}$, Waldir Nagel Schirmer ${ }^{2}$, Alexandre Rodrigues Cabral ${ }^{3}$, Cléverson Luiz Dias Mayer ${ }^{4}$, \\ Pedro Henrique Mildemberger Eurich ${ }^{5}$, Eduardo Henrique Martins ${ }^{6}$ \\ 1, 2, 3, 4, 5, 6 Universidade Estadual do Centro Oeste, Irati, Paraná, Brasil - bianekjulia@gmail.com, \\ wanasch@yahoo.com.br,alexandre.cabral@usherbrooke.ca,engamb.mayer@gmail.com, \\ pedro@recitechambiental.com.br\&edu_hmartins@yahoo.com.br
}

\section{RESUMO}

O aproveitamento energético do biogás de aterros sanitários é uma alternativa sustentável para a disposição final de gases residuais. A estimativa teórica da produção desse biogás torna-se uma ferramenta útil para a avaliação de viabilidade e dimensionamento de sistemas de coleta de gás. Os modelos de geração de biogás de aterro mais utilizados atualmente são os baseados em equações de decaimento de primeira ordem. Os modelos LandGEM e IPCC foram utilizados neste trabalho para a estimativa da geração de gases no aterro municipal de Guarapuava (PR, Brasil), onde os principais parâmetros foram escolhidos com base nos dados frequentemente aplicados para cada modelo: valores default sugeridos para as condições locais específicas, para a aplicação do software LandGEM; e cálculo do potencial de geração de metano $\left(L_{0}\right)$ a partir da análise gravimétrica dos resíduos, para a aplicação da equação sugerida pelo IPCC. Estimou-se uma produção total de biogás de $44.466 .711 \mathrm{Nm}^{3}$. ano-1 aplicando LandGEM e $60.080 .906 \mathrm{Nm}^{3}$. ano-1 aplicando IPCC, onde os picos de produção foram observados nos anos de 2021 e 2020, respectivamente. A escolha do modelo mais adequado dependerá dos dados disponíveis e, para maior aproximação de resultados reais, os parâmetros adotados devem ser analisados com cautela, considerando as diferentes condições climáticas e operacionais de cada caso.

PALAVRAS-CHAVE: Aproveitamento energético, Bioenergia, LandGEM; Resíduos sólidos urbanos (RSU).

\begin{abstract}
The energetic use of landfill biogas is a sustainable alternative for the final disposal of waste gases. The theoretical estimation of the production of this biogas becomes an useful tool for evaluating the feasibility and design of gas collection systems sizing. The most widely used landfill biogas generation models are those based on first-orderdecay equations. LandGEM and IPCC models were used in this work to estimate the generation of gases in the municipal landfill of Guarapuava (PR), and the main parameters were chosen based on the frequently applied data for each model: default values suggested for the specific local conditions, for the application of LandGEM software; and calculating the methane generation potential $\left(L_{0}\right)$ from the gravimetric analysis of the residues, for the application of the equation suggested by the IPCC. A total biogas production of $44,466,711 \mathrm{Nm}^{3}$.ano-1 was estimated by applying LandGEM and $60,080,906 \mathrm{Nm}^{3}$.ano-1 using IPCC, whose production peaks were observed in the years 2021 and 2020, respectively. The choice of the most appropriate model will depend on the available data and, for better approximation to real results, the parameters adopted should be analyzed with caution, considering the difference in climatic and operational conditions of each case.
\end{abstract}

KEYWORDS: Bioenergy, Energetic recovery, LandGEM, Municipal solid wastes (MSW). 


\section{INTRODUÇÃO}

O biogás é gerado a partir de processos físicos, químicos e microbianos que ocorrem dentro da biomassa do aterro (CASTILHOS JÚNIOR et al., 2003; SPOKAS et al., 2006). Devido à natureza orgânica da maior parte dos resíduos, são os processos microbianos que regem a geração de gás (DEUBLEIN \& STEINHAUSER, 2008; MACHADO et al., 2009), predominantemente composto por metano $\left(\mathrm{CH}_{4}\right)$ e dióxido de carbono $\left(\mathrm{CO}_{2}\right)$ (IPCC, 2006; SPOKAS et al., 2006; IMRE et al., 2009; ABUSHAMMALA et al., 2011). Estes processos são sensíveis ao ambiente e dependentes de diversos fatores antrópicos e naturais que afetam constantemente a população microbiana, refletindo diretamente na produção do biogás (CASTILHOS JÚNIOR et al., 2003).

Por apresentar alto potencial energético (CASTILHOS JÚNIOR et al., 2003; SPOKAS et al., 2006), o metano tornase um importante subproduto do processo de biodigestão de resíduos sólidos urbanos (RSU) tendo, a partir de seu aproveitamento, uma fonte de energia renovável (AMINI et al., 2012), economicamente viável e ambientalmente correta (FRANQUETO, 2016). Embora metano e dióxido de carbono sejam os principais componentes do biogás de aterro, outros compostos aparecem em menores proporções, como hidrogênio, nitrogênio, oxigênio, etc. ou mesmo em concentrações a nível de traço (como sulfurados e compostos orgânicos voláteis - COV) representando risco à saúde ou mesmo incômodo olfativo à população exposta (TCHOBANOGLOUS et al., 1993; CASTILHOS JÚNIOR et al., 2003; SEPA, 2004; PAGÉ et al., 2007; MAGHANAKI et al., 2013).

O aproveitamento energético do biogás pode atuar como uma alternativa sustentável para a disposição final de gases residuais. Recentemente, o Brasil apresentou suas futuras ações frente à 21ạ Conferência das Partes (COP-21) da Convenção-Quadro das Nações Unidas sobre Mudança do Clima, na qual sujeita a redução de $37 \%$ das emissões de gases de efeito estufa (GEE) até 2025 e 43\% até 2030 - ambas com base nos índices registrados em 2005. O país firmou também um compromisso referente ao aperfeiçoamento da matriz energética nacional, com ampliação de $28 \%$ para $33 \%$ no uso de energias renováveis (eólica, solar, biomassa e biocombustíveis) até 2030, impulsionando a implantação de projetos de Mecanismo de Desenvolvimento Limpo (MDL) (BRASIL, 2016).

A modelagem matemática possibilita estimar o potencial de geração de biogás no aterro a partir da massa de resíduos depositada. Além de prever as emissões de GEE, essa estimativa possibilita o dimensionamento de sistemas de coleta de biogás a serem implantados no aterro (MACHADO et al., 2009). Existem vários modelos disponíveis, os quais podem ser utilizados para estimar a taxa de geração de biogás ao longo do tempo (BOGNER et al., 1997). Em comparação com outros métodos (como os métodos de campo), os modelos teóricos apresentam vantagens, como o baixo custo e resultados rapidamente observáveis (ABRELPE, 2013).

Os modelos de geração de gás de aterro mais utilizados atualmente são os baseados em equações de decaimento de primeira ordem (MACHADO et al., 2009), que podem ser aplicados utilizando dados específicos de cada local para gerar os parâmetros necessários na estimativa das emissões ou, caso não haja dados disponíveis, utilizando um conjunto de valores-padrão (default) (USEPA, 2005; IPCC, 2006). Modelos de primeira ordem incorporam o efeito do tempo na degradabilidade dos insumos (MACIEL, 2009), assumindo que a decomposição dos resíduos segue a cinética de primeira ordem (WORLD BANK, 2004).

As principais variáveis empregadas nos modelos de primeira ordem são: potencial de geração (Lo), que depende unicamente do tipo de composição do resíduo; e a constante de geração de $\mathrm{CH}_{4}(\mathrm{k})$, que varia em função da umidade, temperatura, $\mathrm{pH}$, tipo de resíduo, disposição de nutrientes para o processo anaeróbio, influenciando na velocidade de geração do biogás (USEPA, 2005; IPCC, 2006; LAGOS et al., 2017). Estes modelos são atualmente recomendados pela Agência de Proteção Ambiental dos Estados Unidos (USEPA, 2005) e pela Agência Intergovernamental de Painel sobre Mudanças Climáticas (IPCC, 2006) para o cálculo de emissões provenientes de aterros sanitários (MACHADO et al., 2009).

Neste contexto, o presente trabalho tem como objetivo estimar o potencial de geração de biogás do aterro sanitário municipal de Guarapuava (PR), aplicando o software LandGEM da USEPA e a equação de decaimento de primeira ordem do IPCC, utilizando como principais parâmetros valores default e dados locais do aterro, respectivamente, com base nos dados frequentemente aplicados para cada modelo, comparando os resultados encontrados.

\section{MATERIAL E MÉTODOS}

\section{Caracterização da área de estudo}

O estudo realizado foi fundamentado em dados 
referentes ao aterro sanitário municipal de Guarapuava PR, localizado na região centro-sul do Estado do Paraná, terceiro planalto, a aproximadamente $260 \mathrm{~km}$ de distância da capital Curitiba. O município está localizado entre as coordenadas de Latitude Sul: $25^{\circ} 23^{\prime} 26^{\prime \prime}$ Sul; e Longitude Oriental: $51^{\circ} 27^{\prime} 15^{\prime \prime}$ Oeste, com extensão territorial de $3.177 .598 \mathrm{Km}^{2}$ e altitude de $1.120 \mathrm{~m}$ (GUARAPUAVA, 2011; GUARAPUAVA, 2016).

O município possui um clima moderado, subtropical, úmido. A temperatura média anual é de $16,8^{\circ} \mathrm{C}$, onde a média máxima é $36^{\circ} \mathrm{C}$ e a mínima, $6,8^{\circ} \mathrm{C}$ (GUARAPUAVA, 2016). Segundo a classificação climática de Köppen, a região de Guarapuava se caracteriza por apresentar clima Cfb (Clima temperado propriamente dito) (IAPAR, 2016), apresentando uma média anual de chuvas de aproximadamente 1685 mm.ano-1 (SALTON et al., 2016).

Segundo dados do IBGE, sua população residente conforme o Censo 2010 era de 167.328 habitantes, sendo estimada uma população de 179.256 habitantes para o ano de 2016 (IBGE, 2016), dos quais aproximadamente 177.820 habitantes são atendidos pela coleta regular de resíduos domiciliares (SNIS, 2015).

O aterro sanitário de Guarapuava iniciou suas atividades em 2011 e hoje recebe aproximadamente 100 toneladas de resíduos sólidos diariamente (GUARAPUAVA, 2011). Destes, aproximadamente $40 \%$ são resíduos orgânicos, $38 \%$ recicláveis e apenas $22 \%$ de rejeito (RECITECH, 2013). Foi projetado com uma vida útil de aproximadamente 9 anos, sendo que seu encerramento está previsto para o ano de 2020 (GUARAPUAVA, 2011).

\section{Estimativa da geração de biogás}

Os modelos LandGEM e IPCC foram utilizados neste estudo para a estimativa da geração de gases em aterro Municipal.

\section{Modelo LandGEM (software da USEPA)}

O modelo LandGEM 3.02 - Landfill Air Emissions Model é um software desenvolvido pela Environmental Protection Agency (EPA), considerado padrão no setor de gases de aterro sanitário, em aterros regulados pela agência, nos Estados Unidos (ABRELPE, 2013), recomendado para a elaboração de projetos e inventários de emissão de biogás e metano em aterros e locais de disposição final (FIRMO, 2013). O modelo matemático utilizado na configuração do software estima a produção de gases a partir da Equação 1 (USEPA, 2005; ABRELPE,
2013).

$$
Q_{C H 4=\sum_{i=1}^{n} 2 k L_{0} M_{i}\left(e^{-k t_{i}}\right)}
$$

Em que:

$Q_{C H 4}=$ taxa de fluxo máximo esperado da geração de biogás de aterro $\left(\mathrm{m}^{3}\right.$.ano $\left.{ }^{-1}\right)$;

$\sum_{i=1}^{n}=$ soma do ano de abertura $+1(i=1)$ até o ano de projeção $(n)$;

$k=$ taxa constante de geração de $\mathrm{CH}_{4}\left(\right.$ ano $\left.^{-1}\right)$;

$L_{0}=$ potencial de geração de metano $\left(\mathrm{m}^{3} \mathrm{Mg}^{-1}\right)$;

$M_{i}=$ massa de resíduos sólidos disposta no primeiro ano $i$ (Mg); e

$t_{i}=$ idade dos resíduos dispostos no primeiro ano $i$ (anos).

\section{Modelo IPCC}

Para a comparação, foi utilizado o modelo do Painel Intergovernamental de Mudanças Climáticas (IPCC), cuja metodologia é sugerida, por exemplo, pela Companhia Ambiental do Estado de São Paulo (CETESB), na elaboração de inventários de emissões de GEE (CETESB, 2009).

Sua abordagem implica na determinação de LO, a partir do teor de carbono orgânico degradável (DOC) dos resíduos. No caso dos resíduos sólidos urbanos, é estimado usando informações sobre os diferentes tipos de resíduos (alimentos, papel, madeira, têxteis, etc.) que compõem a massa do aterro (IPCC, 2006). A Equação 2 é aplicada no modelo (IPCC, 2001; ABRELPE, 2013):

$$
\begin{gathered}
Q_{C H 4}=\sum x\left\{\left[\left(A \cdot k \cdot R S U T_{(x)} \cdot \operatorname{RSUF}_{(x)} \cdot L_{0(t)}\right) \cdot e^{-k \cdot(t-x)}\right]\right. \\
\left.-R_{x}\right\} \cdot(1-O X)
\end{gathered}
$$

Em que:

$Q_{C H 4}=$ metano gerado ao ano $\left(\mathrm{Gg}_{\mathrm{CH} 4 . \mathrm{ano}^{-1}}\right)$;

$A=$ fator de normalização para a soma (adimensional);

$A=\frac{1-e^{-k}}{k}$;

$k=$ taxa constante de geração de $\mathrm{CH}_{4}\left(\mathrm{ano}^{-1}\right)$;

$R S U_{T(x)}=$ quantidade total de resíduo sólido urbano gerado no ano $x\left(G_{\text {RSu.ano }}{ }^{-1}\right)$;

$R S U_{F(x)}=$ fração de RSU destinado ao aterro no ano $x$ (adimensional);

$L_{0(t)}=$ potencial de geração de metano $\left(\mathrm{Gg}_{\mathrm{CH} 4} \cdot \mathrm{Gg}_{\mathrm{RSU}}{ }^{-1}\right)$;

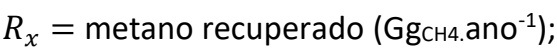

$T=$ ano do inventário (ano); e

$x=$ anos os quais os dados foram considerados. 


$$
L_{0(t)}=M C F_{(t)} \cdot D O C_{(t)} \cdot D O C f \cdot F \cdot 16 / 12
$$

Em que:

$M C F_{(t)}=$ fator de correção do metano referente ao gerenciamento dos locais de destinação (adimensional); $D O C_{(t)}=$ carbono orgânico degradável $\left(\mathrm{g}_{\mathrm{C}} \cdot \mathrm{g}_{\mathrm{RSU}}{ }^{-1}\right)$; DOCf = fração do DOC que decompõe (adimensional); $F=$ fração de metano no biogás (adimensional); $16 / 12$ = razão de conversão de carbono $(C)$ a metano $\left(\mathrm{CH}_{4}\right)$ (adimensional); e

$\mathrm{DOC}_{(\mathrm{t})}=$ somatória das frações de composição do resíduo.

O fator de Oxidação (OX) expressa a quantidade de metano da massa residual que é oxidada no solo e na camada de cobertura, sendo uma medida adimensional (IPCC, 2006).

\section{Estimativa da geração de resíduos}

A estimativa de geração de resíduos deu-se a partir de regressão exponencial, com base em dados reais de disposição dos resíduos no aterro, desde o ano de sua abertura até o ano de 2016, gerando a Equação $3\left(R^{2}=\right.$ $0,75)$, com a qual foram calculados os valores equivalentes aos anos de 2017 a 2020:

$$
Q_{C H 4=\sum_{i=1}^{n} 2 k L_{0} M_{i}\left(e^{-k t_{i}}\right)}
$$

Em que:

$x=$ Ano de deposição; e

$y=$ Quantidade de resíduo estimada $(\mathrm{t})$.

Seleção das variáveis

Na aplicação do software LandGEM, as variáveis $k$ e $L_{0}$ adotadas foram os valores default estabelecidos pelo programa, indicados para a regulamentação da Clean Air Act (CAA), dos Estados Unidos, que para climas mais úmidos (com precipitações de, no mínimo, $635 \mathrm{~mm}$ anuais) recomenda um $\mathrm{k}$ de $0,05 \mathrm{ano}^{-1}$ e $\mathrm{L}_{0}$ de $170 \mathrm{~m}^{3} . \mathrm{Mg}^{-1}$ (USEPA, 2005). Os valores referentes à quantidade de resíduos disposta no aterro são dados reais, desde o ano de início das atividades, e calculados a partir de uma estimativa (descrita no item 2.3), a partir do ano de 2017.

Para a aplicação da equação do IPCC, o valor de $L_{0}$ (potencial de geração de metano) foi obtido a partir da entrada de dados reais do aterro (análise gravimétrica), calculado através da equação 2.1 resultando em um valor de $134,12 \mathrm{Nm}^{3}{ }_{\mathrm{CH}} \cdot \mathrm{t}_{\mathrm{RSU}}{ }^{-1}$.
Os demais parâmetros foram selecionados seguindo a metodologia proposta pelo IPCC (IPCC, 2006) considerando as particularidades do local de estudo, em que:

$\mathrm{k}=0,05 \mathrm{ano}^{-1}$, valor recomendado pelo IPCC para locais com temperatura média anual menor que $20^{\circ} \mathrm{C}$, para clima boreal e temperado;

$M C F_{(t)}=1,0$, valor recomendado para aterros sanitários; DOC $f=0,5$, considerando que alguns resíduos não se decompõem, ou sua taxa de decomposição é muito lenta; $\mathrm{F}=0,5$ pois pressupõem-se que $50 \%$ do biogás formado é composto por metano; e

$\mathrm{R}_{x}$ e OX são considerados nulos.

O modelo matemático sugerido pelo IPCC possibilita calcular exclusivamente o volume de metano emitido pelo aterro, permitindo contabilizar a recuperação do gás metano obtido, e introduzir um fator de oxidação do gás na camada superficial (IPCC, 2006). No caso do aterro de Guarapuava, o fator de oxidação (OX) e a recuperação de metano $\left(R_{x}\right)$ foram considerados nulos, admitindo-se a inexistência de sistemas de recuperação e devido à ausência de dados de campo referentes à oxidação do gás na camada de cobertura, uma vez que o objetivo do trabalho é avaliar o potencial de produção de biogás, ao invés de emissões para a atmosfera.

\section{RESULTADOS E DISCUSSÃO}

Dois modelos de primeira ordem foram aplicados para a previsão da taxa de geração de metano do aterro municipal de Guarapuava (LandGEM e IPCC). A Figura 1 mostra a produção anual do gás de aterro prevista por cada modelo.

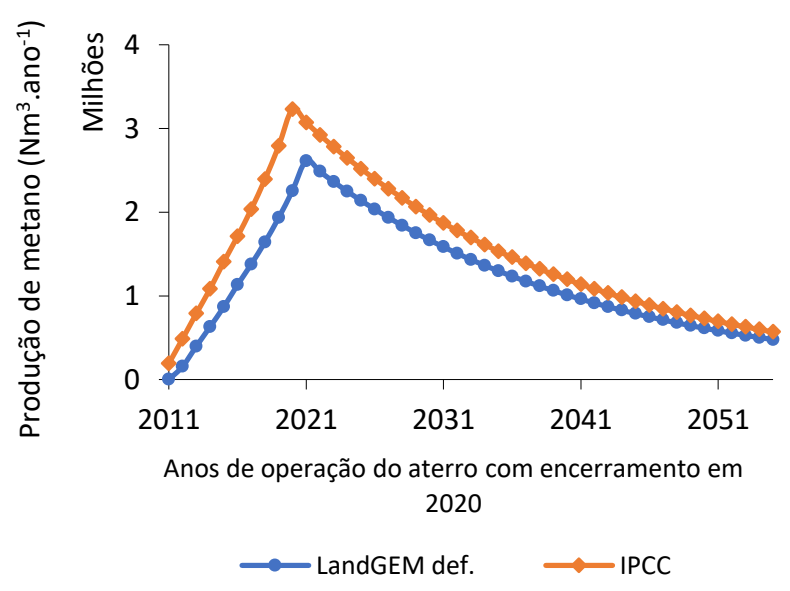

Figura 1. Estimativa da produção de metano no aterro municipal de Guarapuava. 
O uso do LandGEM possibilitou avaliar a produção de metano, gás carbônico e outros gases, obtendo-se um total de biogás acumulado, entre os anos 2011 e 2025, de 44.466.711 $\mathrm{Nm}^{3}$.ano-1, sendo 22.233.355 $\mathrm{Nm}^{3}$ o volume de metano acumulado, uma vez que o modelo prediz que o volume de gás carbônico se iguala ao de metano, e que os outros gases apresentam um montante pouco significativo.

Novamente, considerando a igualdade entre as proporções dos principais gases que compõem o biogás (como o metano e o dióxido de carbono), obteve-se a partir da aplicação da metodologia IPCC, um total de biogás acumulado entre os anos 2011 e 2025 de 60.080.906 $\mathrm{Nm}^{3}$.ano-1, sendo $30.040 .453 \mathrm{Nm}^{3}$ o volume de metano acumulado.

A metodologia IPCC apresenta valores de produção total de biogás maiores que os obtidos pelo software LandGEM, mesmo utilizando um potencial de geração menor (Lo). A diferença observada entre os resultados pode ser justificada pelas diferentes variáveis consideradas em cada modelo. Assim, recomendam-se estudos que abordem as particularidades do aterro em questão, de modo a complementar e otimizar os valores obtidos.

Segundo Scharffe \& Jacobs (2006) uma desvantagem do modelo utilizado pelo software LandGEM dá-se pelo fato de não se considerar as diferenças na composição do conteúdo da massa do aterro. No entanto, em casos onde não existem informações suficientes para servirem de insumo a outros modelos (como o IPCC), a aplicação do LandGEM pode ser vantajosa.

A produção máxima de metano para o LandGEM foi de 2.612.322 $\mathrm{Nm}^{3} \mathrm{CH}_{4}$.ano ${ }^{-1}$ e $3.227 .394 \mathrm{Nm}^{3} \mathrm{CH}_{4}$.ano ${ }^{-1}$ para IPCC, sendo que esses valores de produção foram atingidos no ano 2021, um ano após o previsto encerramento do aterro, e em 2020, ano do encerramento, respectivamente, mantendo-se a mesma constante de degradação $k$, que define a velocidade de degradação dos resíduos e a consequente geração de biogás (FIRMO, 2013) para ambos os modelos.

Mesmo sendo equações cinéticas de primeira ordem e considerando parâmetros de entrada semelhantes, foram observados resultados distintos nos picos de produção para cada modelo. A produção máxima foi observada em anos diferentes para cada modelo, o que se deve ao fato de o LandGEM considerar que a emissão de metano começará somente após o fim do primeiro ano de deposição da massa de resíduos (SILVA, 2012; ABRELPE, 2013), diferentemente do modelo do IPCC, que considera que a emissão de metano começa ao longo do primeiro ano de deposição.

A curva de geração de metano deste último modelo é deslocada para a esquerda no eixo do tempo (abscissa) do gráfico e, concomitantemente, ocorre o deslocamento equivalente do ápice de produção de metano. Assim, verifica-se que o ápice de geração de biogás ocorrerá, provavelmente, quando o máximo de resíduos estiver disposto no aterro, ao final da sua vida útil.

\section{CONCLUSÕES}

O modelo IPCC estimou uma produção total de biogás de 60.080.906 Nm $\mathrm{Nmo}^{3}$. ${ }^{-1}$, para o ano de 2025, sendo $30.040 .453 \mathrm{Nm}^{3}$ o volume de metano acumulado, enquanto o software LandGEM apresentou uma estimativa mais conservadora, com um total de produção de 44.466.711 Nm $\mathrm{Nmo}^{3}$. an, sendo 22.233.355 $\mathrm{Nm}^{3}$ o volume de metano acumulado. Os picos de produção foram no ano de 2020 para a metodologia IPCC e 2021 para o LandGEM, alcançando 3.227.394 $\mathrm{Nm}^{3} \mathrm{CH}_{4}$.ano-1 e 2.612.322 $\mathrm{Nm}^{3} \mathrm{CH}_{4}$.ano-1 ${ }^{-1}$, respectivamente.

$\mathrm{Na}$ utilização de modelos matemáticos para a estimativa de produção de biogás em aterros sanitários, os parâmetros adotados e as considerações a serem feitas devem ser avaliadas com cautela, devido às diferentes condições climáticas e operacionais de cada caso, tendo em vista que esse tipo de estimativa é um importante pressuposto para avaliação de viabilidade e dimensionamento de sistemas de recuperação de energia a partir de resíduos.

\section{AGRADECIMENTOS}

Os autores agradecem à Coordenação de Aperfeiçoamento de Pessoal de Nível Superior (CAPES), no âmbito do Ciência Sem Fronteiras (CsF), pelo suporte financeiro ao projeto, e à Secretaria Municipal de Meio Ambiente de Guarapuava ( $P R$ ), pelo apoio técnico ao trabalho.

\section{REFERÊNCIAS}

ABRELPE - ASSOCIAÇÃO BRASILEIRA DE EMPRESAS DE LIMPEZA PÚBLICA E RESÍDUOS ESPECIAIS. Atlas brasileiro de emissões de gases de efeito estufa e potencial energético na destinação de resíduos sólidos. São Paulo, 2013. 171 p.

ABUSHAMMALA, M. F. M.; BASRI, N. E. A.; BASRI, H.; EL-SHAFIE, A. H.; KADHUM, A. A. H. Regional landfills methane emission inventory in Malaysia. Waste Management \& Research, v. 29, n. 8, p. 863-873, 2011. 
AMINI, H. R.; REINHART, D. R.; MACKIE, K. R. Determination of first-order landfill gas modeling parameters and uncertainties. Waste Management, v.32, n. 2, p. 305-316, 2012.

BOGNER, J.; HICKMAN, H. L.; HUITRIC, R.; LIPPY, S. G.; PACEY, J.; ROQUETA, A.; WILES, C. Final report: comparison of models for predicting landfill methane recovery. In: DRIVE, R. B.; AUGENSTEIN, D. The solid waste association of North America, 1997, p. 1-5.

BRASIL. Informação adicional sobre a IDNC apenas para fins de esclarecimento. 2016. Disponível em: <http://www.itamaraty. gov.br/images/ed desenvsust/BRASIL-iNDC-portugues.pdf>.

Acesso em: 02/06/ 2016.

CASTILHOS JÚNIOR, A. B. de.; MEDEIROS, P. A.; FIRTA, I. N.; LUPATINI, G.; SILVA, J. D. da. Principais processos de degradação de resíduos sólidos urbanos. In: CASTILHOS JÚNIOR, A. B. de; (Ed.). Resíduos sólidos urbanos: aterro sustentável para municípios de pequeno porte. Rio de Janeiro: ABES, 2003. p. 1950 .

CETESB - COMPANHIA AMBIENTAL DO ESTADO DE SÃO PAULO. Guia para a elaboração de inventários corporativos de emissões de Gases do Efeito Estufa (GEE). São Paulo: FGV, 2009. $22 \mathrm{p}$.

DEUBLEIN, D.; STEINHAUSER, A. Biogas from waste and renewable resources: an introduction. Weinheim: Wiley- $\mathrm{VCH}$, 2008. $469 \mathrm{p}$.

FIRMO, A. L. B. Estudo numérico e experimental da geração de biogás a partir da biodegradação de resíduos sólidos urbanos. 2013. 286 f. Tese (Doutorado em Engenharia Civil) Universidade Federal de Pernambuco, Recife, 2013.

FRANQUETO, R. Avaliação de emissões fugitivas de biogás de aterro sanitário em camada de cobertura convencional e com características melhoradas aplicando método de câmara de fluxo em regime estático. 2016. 93 f. Dissertação (Mestrado em Engenharia Sanitária e Ambiental) - Universidade Estadual do Centro-Oeste, Irati, 2016.

GUARAPUAVA. Plano municipal de saneamento básico Município de Guarapuava-PR. Guarapuava, 2011. 151 p. Disponível em: <http://www.guarapuava.pr.gov.br/wp-content/ uploads/pms-guarapuava-verfinal.pdf>. Acesso em: 02/02/2017.

GUARAPUAVA. Sobre Guarapuava, 2016. Disponível em: <http://www.guarapuava.pr.gov.br/turista/sobre-guarapuava>. Acesso em: 02/12/2016.

IAPAR - INSTITUTO AGRONÔMICO DO PARANÁ. Cartas climáticas do Paraná: classificação climática segundo Köppen. IAPAR, 2016. Disponível em: <http://www.iapar.br/ modules/conteudo/conteudo.php?conteudo $=597>$. Acesso em: 02/12/2016.

IBGE - INSTITUTO BRASILEIRO DE GEOGRAFIA E ESTATÍSTICA. IBGE cidades. IBGE, 2016. Disponível em: <https://cidades.ibge.gov.br/v4/brasil/pr/guarapuava/panoram a> . Acesso em: 15/01/2017.

IMRE, E.; KOVÁCS, K. L.; BAGI, Z.; ÁCS, N.; PEREI, R. K.; BARTHA,
I.; TRANG P. Q.; TELEKES, G. Biotechnological methods to increase landfill gas production. In: Proceedings Sardinia 2009: International Waste Management and Landfill Symposium, 12., Anais..., Cagliari, CISA, 2009, p. 5-9.

IPCC - INTERGOVERNMENTAL PANEL ON CLIMATE CHANGE. Good practice guidance and uncertainty management in national greenhouse gas inventories. 2001. Disponível em: $<$ http://www.ipcc-nggip.iges.or.jp/public/gp/english/5 Waste. pdf>. Acesso em: 18/09/2017.

IPCC - INTERGOVERNMENTAL PANEL ON CLIMATE CHANGE. Solid waste disposal. Guidelines for National Greenhouse Gas Inventories, v. 5, p. 1-40, 2006.

LAGOS, D. A.; HÉROUX, M.; GOSSELIN, R.; CABRAL, A. C. Optimization of a landfill gas collection shutdown based on an adapted first-order decay model. Waste Management, v. 63, p. 238-245, 2017.

MACHADO, S. L.; CARVALHO, M. F.; GOURC, J-P.; VILAR O. M.; NASCIMENTO, J. C. F. Methane generation in tropical landfills: Simplified methods and field results. Waste Management, v. 29, p. 153-161, 2009.

MACIEL, F. J. Geração de biogás e energia em aterro experimental de resíduos sólidos urbanos. 2009. $333 \mathrm{f}$. Tese (Doutorado em Engenharia Civil) - Universidade Federal de Pernambuco, Recife, 2009.

MAGHANAKI, M. M.; GHOBADIAN, B.; NAJAFI, G.; GALOGAH, R. J. Potential of biogas production in Iran. Renewable and Sustainable Energy Reviews, v. 28, p. 702-714, 2013.

PAGÉ, T.; NARJOUX, A.; GUY, C.; CARON, R. F.; FÉCIL, B. Impact evaluation of landfills on air quality: odors and toxic compounds. In: International Waste Management and Landfill Symposium, 11., Anais..., Cagliari, 2007.

RECITECH. Diagnóstico da coleta dos resíduos sólidos urbanos com ênfase no levantamento de custos. Guarapuava, 2013. 125 p.

SALTON, F. G.; MORAIS, H.; CARAMORI, P. H.; BORROZZINO, E. Climatologia dos episódios de precipitação em três localidades no Estado do Paraná. Revista Brasileira de Meteorologia, v. 31, n. 4, p. 626-638, 2016.

SCHARFF, H.; JACOBS, J. Applying guidance for methane emission estimation for landfills. Waste Management, v. 26, p. 417-429, 2006.

SEPA - SCOTTISH ENVIRONMENT PROTECTION AGENCY. Guidance for monitoring enclosed landfill gas flares. Technical Report P1-405, 2004. 74 p.

SILVA, T. R. Metodologia para a determinação teórica da potência ótima conseguida a partir da combustão do biogás gerado em aterro sanitário: Estudo de caso do aterro sanitário de Itajubá-MG. 2012. 160 f. Dissertação (Mestrado em Engenharia de Energia) - Universidade Federal de Itajubá, Itajubá, 2012.

SNIS - SISTEMA NACIONAL DE INFORMAÇÃO SOBRE 
SANEAMENTO. Série histórica, 2015: informação e indicadores municipais consolidados. 2015. Disponível em: $<$ http://app.cidades.gov.br/serieHistorica/>. Acesso em: $15 / 01 / 2017$.

SPOKAS, K.; BOGNER, J.; CHANTON, J. P.; MORCET, M.; ARAN, C.; GRAFF, C.; MOREAU-LE GOLVAN, Y.; HEBE, I. Methane mass balance at three landfill sites: What is the efficiency of capture by gas collection systems? Waste Management, v. 26, p. 516525, 2006.

TCHOBANOGLOUS, G.; THEISEN, H.; VINIL, S. Integrated solid waste management. Engineering principles and management issues. New York: McGraw-Hill. 1993, 978 p.

USEPA - UNITED STATES ENVIRONMENTAL PROTECTION AGENCY. First-order kinetic gas generation model parameters for wet landfills (EPA-600/R-05/072). Washington, 2005. 66 p.

WORLD BANK. Handbook for the preparation of landfill gas to energy projects in Latin America and the Caribbean. ESMAP (Energy Sector Management Assistance Programme), v. 651, p. 6-52, 2004.

Recebido em 25/10/2017

Aceito em 12/12/2017 